\title{
Editorial for JETC Special Issue on Alternative Computing Systems
}

"Error is viewed, not as an extraneous and misdirected or misdirecting accident, but as an essential part of the process.... Our present treatment of error is unsatisfactory and ad hoc." -J. von Neumann (1956).

Well before 1956, John von Neumann recognized and voiced repeatedly his conviction that handling errors is a critical aspect of computing. For over 50 years, the field of computing has relied on powerful methods such as defect minimization, design margining, abstraction, redundancy, backtracking, and virtualization at the technology, circuits, architecture, and software levels to avoid errors and exploit the scaling enabled by Moore's Law. However, a drastic shift in the computing model may be due, as traditional technology scaling itself poses risks to the continued advancement of computers; the memory gap is becoming more and more important, power is continuing to be a limiting factor, and variation- and reliability-induced errors in nanoscale technologies are getting more difficult to contain.

Recent research has looked into new computing models as a solution. These have particular relevance in the context of emerging applications, which are rapidly gaining prominence. Instead of focusing on exact computations from traditional input sources, more diverse inputs sources, often based on ensembles (of sensors, users, other computers), are enabling probabilistic or approximate models for computing towards high-level inferences. These models have several implications, such as reduced power or latency, particularly when they are considered in the context of emerging technologies. Generally speaking, such computing models have so far been shown to be applicable only in specific cases; however, broader benefits are also possible. In brain-inspired computing, as an example, while substantial background including the decades of research in neural networks exists, recent advancements focus on general-purpose computing and emerging machine-learning methods.

While it is possible to study these computing models independent of applications, designing new computing models in a way that is explicitly driven by these upcoming applications would lead to more systematic and more substantial advances. Further, it will also enable directed efforts on the application levels that are influenced by these computing models. With these goals, we have organized an ACM JETC Special Issue where researchers from alternative computing, system design, and emerging applications areas could publish their insights towards the common goal of improving computer system designs.

For this special issue, we received 12 submissions. After two rounds of reviews, nine papers have been selected for the special issue. The papers are organized into the following groups: building blocks, architecture, and biologically inspired systems. Each group contains one invited paper, each of which has also gone through a review process of similar scrutiny as the regular papers.

We start the special issue with "Trading accuracy for energy in stochastic circuit design" by Alaghi et al. The authors of this invited paper introduce a path balancing technique that limits accuracy loss. In "Design of approximate compressors for multiplication," Gorantla and Deepa design Dadda-style multipliers utilizing a new compressor design. In Computing Polynomials using Unipolar Stochastic Logic," Liu

2017 Copyright is held by the owner/author(s).

1550-4832/2017/03-ART38

DOI: http://dx.doi.org/10.1145/3022700

ACM Journal on Emerging Technologies in Computing Systems, Vol. 13, No. 3, Article 38, Publication date: March 2017. 
and Parhi implement stochastic subtraction and factorization without need to use the more commonly utilized Bernstein polynomials. Finally, in "Sketching Computation with Stochastic Processing Engines," Alawad and Lin utilize probability density functions to gradually improve accuracy of computation and demonstrate its practicality using an FPGA implementation.

Starting the second category of papers, Britt and Humble, in their work titled "Highperformance computing with quantum processing units," introduce the notion of utilizing quantum processors as co-processors, and work out the architectural details of co-integrating them with existing high-performance computing systems. In the invited paper "PPU: A control error-tolerant processor for streaming applications with formal guarantees," Golnari et al. introduce a low-overhead error-tolerant processor that works by converting fatal control errors to tolerable data errors. In "Mobile unified memory-storage structure based on hybrid non-volatile memories," Yoon et al. combine the traditional dynamic random access with the more recent non-volatile memory to form a hybrid memory region that boosts buffering and power performance.

Starting the bio-inspired category of papers, Guha et al. target security improvement against hardware trojan horses with their biology-inspired work titled "Real time SoC security against passive threats using crypsis behaviour of geckos." In the invited paper "Toward human-scale brain computing using 3D wafer scale integration," Kumar et al. implement a primate-scale brain on chip using 3D wafer-scale integration technology.

Even this one special issue shows that the research vectors associated with alternative computing are numerous. In fact, if such an approach to computing is to prevail many further issues, they must be brought to the forefront and studied. We hope this issue contributes a positive step towards this goal.

\author{
Rasit O. Topaloglu \\ IBM \\ Naveen Verma \\ Princeton University
}

Guest Editors 